\title{
JOINT SPECTRA AND ANALYTIC SET-VALUED FUNCTIONS
}

\author{
BY
}

\author{
M. KLIMEK
}

\begin{abstract}
We investigate analyticity of joint spectra of $A^{m}$-valued holomorphic mappings, where $A$ denotes a complex Banach algebra. We show also that if $K$ is an analytic set-valued function whose values are compact subsets of $\mathbf{C}^{n}$ and $d$ is the transfinite diameter in $\mathbf{C}^{n}$, then the upper-semicontinuous regularization of $\log d(K)$ is plurisubharmonic. Moreover, we give higher dimensional extensions of Aupetit's Scarcity Theorem.
\end{abstract}

1. Introduction. Analytic functions whose values are compact subsets of the plane were introduced by Oka [9] in 1934. He defined them by means of pseudoconcavity. Afterwards they were practically forgotten for nearly fifty years. In 1980, Słodkowski [15] proposed a different definition of analytic set-valued functions using plurisubharmonic functions. This new definition was equivalent to the old one in the case of functions with values contained in $\mathbf{C}$ and was suitable for higher dimensions. Moreover it was much more flexible. The publication of [15] seems to have been the turning point in the history of analytic set-valued (or multivalued) functions. In the 1980's numerous articles dealing with this topic have already appeared. They were written by various authors including Alexander, Aupetit, Ransford, Słodkowski, Vesentini, Wermer, Zemanek and Zraibi. Ransford's dissertation [10] is an excellent survey article that also contains plenty of new results.

Analytic set-valued functions are very useful in spectral theory. A deep result proved in [15] says that if $f$ is a holomorphic function with values in a Banach algebra, then the spectrum of $f(z)$ is an analytic set-valued function with respect to $z$. (Moreover every analytic set-valued function with values contained in $\mathbf{C}$ is locally of this form (see [15] for details).)

In our first theorem we prove a similar property for joint spectra. Let $A$ be a complex Banach algebra with unit $e$. Following Harte [4] we define for $a=$ $\left(a_{1}, \ldots, a_{m}\right) \in A^{m}$ the left joint spectrum of $a$,

$$
\sigma_{A}^{L}(a)=\left\{z \in \mathbf{C}^{m}: e \notin \sum_{j} A\left(a_{j}-z_{j} e\right)\right\},
$$

the right joint spectrum of $a$,

$$
\sigma_{A}^{R}(a)=\left\{z \in \mathbf{C}^{m}: \epsilon \notin \sum_{j}\left(a_{j}-z_{j} e\right) A\right\},
$$

Received by the editors February 5, 1985.

1980 Mathematics Subject Classification. Primary 46H99, 32F05; Secondary 32H99, 47A10. 
and the joint spectrum of $a$,

$$
\sigma_{A}(a)=\sigma_{A}^{L}(a) \cup \sigma_{A}^{R}(a) .
$$

Throughout the paper ` will denote the polynomially convex hull.

THEOREM 1. Let $\Omega$ be an open subset of $\mathbf{C}^{n}$ and let $A$ be a complex Banach algebra with unit. Let $f=\left(f_{1}, \ldots, f_{m}\right): \Omega \rightarrow A^{m}$ be a holomorphic mapping such that $\left\{f_{1}(z), \ldots, f_{m}(z)\right\}$ is a commuting system of elements for each $z \in \Omega$. Then $z \rightarrow \hat{\sigma}_{A}(f(z))$ is an analytic set-valued function on $\Omega$. Furthermore, if $A$ is commutative, then $z \rightarrow \sigma_{A}(f(z))$ is analytic.

This theorem together with the maximum principle for analytic set-valued functions (see e.g. $[\mathbf{1 0}]$ ) implies the maximum principle for the polynomially convex hulls of joint spectra proved by Vesentini $[18$, Theorem I].

Yamaguchi [20] has proved that if $z \rightarrow K(z)$ is an analytic set-valued function with values in Comp $\mathbf{C}$, then $z \rightarrow \log c(K(z))$ is plurisubharmonic (where $c=$ logarithmic capacity $=$ Fekete's transfinite diameter $)$. In the next theorem we show that this result can be generalized for analytic functions with values in Comp $\mathbf{C}^{m}$ provided that Fekete's transfinite diameter is replaced by the $\mathbf{C}^{m}$-transfinite diameter (for $m=1$ they coincide). It should be noted that Yamaguchi's theorem can also be extended in a different direction (see $[\mathbf{1 8}]$ ).

We shall prove the following

THEOREM 2. Let $\Omega \subset \mathbf{C}^{n}$ be open and let $K: \Omega \rightarrow \operatorname{Comp} \mathbf{C}^{m}$ be an analytic set-valued function. If $d$ is the transfinite diameter in $\mathbf{C}^{m}$, then the function $u: \Omega \rightarrow[-\infty, \infty)$ defined by the formula $u(z)=(\log d(K(z)))^{*}$ is plurisubharmonic (* stands for the upper semicontinuous regularization). Moreover if $\Omega$ is connected, then either $K(z)$ is pluripolar for all $z$ or the set $\{z \in \Omega: K(z)$ is pluripolar $\}$ is itself pluripolar.

Aupetit $[\mathbf{1}, \mathbf{2}]$ has proved the so-called 'Scarcity Theorem' that gives a classification of analytic functions of one variable whose values are finite subsets of the complex plane (see also [10, Theorem 3.11]). The next two results are higher dimensional extensions of Aupetit's result. Before formulating our theorems, recall that a set $K \subset \mathbf{C}^{m}$ is said to be unisolvent for polynomials of degree $k$ if the following implication is true: If $f: \mathbf{C}^{m} \rightarrow \mathbf{C}$ is a polynomial of degree $\leq k$ and $f=0$ on $K$, then $f=0$ in $\mathbf{C}^{n}$.

THEOREM 3. Let $\Omega$ be an open connected subset of $\mathbf{C}^{n}$ and let $K: \Omega \rightarrow$ Comp $\mathbf{C}^{m}$ be an analytic set-valued function. Define ord $K(z)=\sup \{k \geq 0: K(z)$ is unisolvent for polynomials of degree $k\}$ for $z \in \Omega$.

Then either $\{z \in \Omega$ : ord $K(z)<\infty\}$ is pluripolar or there exists a positive integer $p$ and a pluripolar subset $F$ of $\Omega$ such that for every $z \in \Omega \backslash F$, ord $K(z)=p$ and for every $z \in F$, ord $K(z)<p$.

THEOREM 4. Let $\Omega$ and $K$ be as in Theorem 3. Then either $\{z \in \Omega: \# K(z)<$ $\infty\}$ is pluripolar or there is a positive integer $p$ and an analytic subvariety $F$ of $\Omega$ such that for each $z \in \Omega \backslash F, \# K(z)=p$ and for each $z \in F, \# K(z)<p$. In the latter case there exists a holomorphic mapping $H: \Omega \times \mathbf{C}^{m} \rightarrow \mathbf{C}^{t}$ (where $t=(m-1) p+1)$ such that $w \in K(z)$ if and only if $H(z, w)=0$. 
It should be emphasized that even for $m=1$ the functions $z \rightarrow \sigma_{A}^{L}(f(z))$ and $z \rightarrow \sigma_{A}^{R}(f(z))$ may not be analytic (see $[\mathbf{1 0}$, p. 83]). In spite of this, Theorems 2, 3 and 4 are true for $z \rightarrow \sigma_{A}^{L}(f(z)), z \rightarrow \sigma_{A}^{R}(f(z))$ and $z \rightarrow \sigma_{A}(f(z))$ provided that $f$ satisfies the assumptions of Theorem 1 . The reason for this is quite simple: For any compact subset $K$ of $\mathbf{C}^{m}, d(K)=d(\hat{K})$ (see [21]), ord $K=\operatorname{ord} \hat{K}$ and, if $K$ is finite, $K=\hat{K}$.

The author wishes to thank Sean Dineen for conversations.

2. Set-valued mappings. Let $X$ and $Y$ be topological spaces. A mapping $F$ that assigns to each point $x$ of $X$ a closed subset $F(x)$ of $Y$ is said to be upper (lower) semicontinuous if for every open (closed) set $S \subset Y$ the set $\{x \in X: F(x) \subset S\}$ is open (closed). In particular a function $u: X \rightarrow[-\infty,+\infty]$ is upper (lower) semicontinuous in the usual sense if and only if the mapping $F_{u}$ defined by $F_{u}(x)=$ $[-\infty, u(x)]$ is upper (lower) semicontinuous in the sense of the above definition (for more details see $[\mathbf{5}, \mathbf{6}, \mathbf{3}])$. Let $\operatorname{Comp} Y$ denote the space of all nonempty compact subsets of $Y$. If $Y$ is a metric space, one can define the Hausdorff metric on Comp $Y$ by the formula

$$
\rho(A, B)=\max \left\{\sup _{a \in A} \operatorname{dist}(a, B), \sup _{b \in B} \operatorname{dist}(b, A)\right\}
$$

for $A, B \in \operatorname{Comp} Y$. The pair (Comp $Y, \rho$ ) is a metric space and a mapping $K: X \rightarrow$ Comp $Y$ is continuous if and only if it is both upper and lower semicontinuous.

Let $\Omega$ be an open subset of $\mathbf{C}^{n}$. A mapping $K: \Omega \rightarrow \operatorname{Comp} \mathbf{C}^{m}$ is said to be analytic set-valued (ASV) if it is upper semicontinuous and the following condition is satisfied:

(ASV) For each open subset $\Omega_{1}$ of $\Omega$ and for each plurisubharmonic function $u$ defined on an open neighbourhood of the set $\left\{(z, w) \in \Omega_{1} \times \mathbf{C}^{m}: w \in K(z)\right\}$ the function $v: \Omega_{1} \rightarrow[-\infty,+\infty)$ is plurisubharmonic, where

$$
v(z)=\sup \{u(z, w): w \in K(z)\} \quad \text { for } z \in \Omega_{1} .
$$

It is clear that (ASV) is a local property.

EXAMPLE 2.1 (RANSFORD [10]). Let $u: \Omega \rightarrow[-\infty,+\infty)$ be an upper semicontinuous function on an open set $\Omega \subset \mathbf{C}^{n}$. Define $K(z)=\{w \in \mathbf{C}:|w| \leq \exp (u(z))\}$. Then $K$ is ASV if and only if $u$ is plurisubharmonic.

EXAMPLE 2.2 (RANSFORD [10]). Let $f: \Omega \rightarrow \mathbf{C}^{m}$ be a function on an open set $\Omega \subset \mathbf{C}^{n}$. Then $K(z)=\{f(z)\}, z \in \Omega$, is ASV if and only if $f$ is holomorphic.

The following generalization of the last example is due to Ransford [10] and Zraibi [22].

THEOREM 2.3. Let $\Omega \subset \mathbf{C}^{n}$ be connected and $K: \Omega \rightarrow \operatorname{Comp}^{m}$ be an analytic set-valued function. If $U$ and $V$ are disjoint open sets in $\mathbf{C}^{m}$ such that $K(z) \subset U \cup V$ for all $z \in \Omega$, then either $K(z) \cap U=\varnothing$ for all $z \in \Omega$ or $K(z) \cap U \neq \varnothing$ for all $z \in \Omega$. In the latter case $z \rightarrow K(z) \cap U$ is an analytic set-valued function.

The next result is due to Słodkowski $[\mathbf{1 5}]$. 
THEOREM 2.4. (a) If $K$ and $L$ are analytic set-valued functions such that the values of $K$ are contained in the domain of $L$, then $L \circ K$ is an analytic set-valued function where $L \circ K(z)=\bigcup\{L(w): w \in K(z)\}$ for any $z$ in the domain of $K$.

(b) If $K$ and $L$ are analytic set-valued functions defined on the same open set $\Omega$, then $K \times L$ is an analtyic set-valued function, where $(K \times L)(z)=K(z) \times L(z)$, $z \in \Omega$.

3. Transfinite diameter in $\mathbf{C}^{n}$. Let $x: \mathbf{N} \rightarrow \mathbf{Z}_{+}^{n}$ be a bijection such that $|x(j)| \leq|x(j+1)|$ for $j \geq 1$, where $|\alpha|=\alpha_{1}+\alpha_{2}+\cdots+\alpha_{n}$ for any multi-index $\alpha=\left(\alpha_{1}, \ldots, \alpha_{n}\right) \in \mathbf{Z}_{+}^{n}$. Define $p_{j}=|x(1)|+|x(2)|+\cdots+\left|x\left(m_{j}\right)\right|$, where $m_{j}$ is the number of monomials in $\mathbf{C}^{n}$ of degree $\leq j$. Clearly $m_{j}=\left(\begin{array}{c}n+j \\ j\end{array}\right)$. Notice that $p_{j}$ does not depend on our choice of $x$.

Let $\xi_{1}, \ldots, \xi_{m}$ be points in $\mathbf{C}^{n}$. Define $e_{j}(\xi)=\xi^{x(j)}$ for all $\xi \in \mathbf{C}^{n}$ and $V\left(\xi_{1}, \ldots, \xi_{m}\right)=\operatorname{det}\left[e_{i}\left(\xi_{j}\right)\right]_{1 \leq i, j \leq n}$. For a compact set $K \subset \mathbf{C}^{n}$ define

$$
V_{m}(K)=\sup \left\{\left|V\left(\xi_{1}, \ldots, \xi_{m}\right)\right|:\left\{\xi_{1}, \ldots, \xi_{m}\right\} \subset K\right\} .
$$

We call the number

$$
d(K)=\limsup _{j \rightarrow \infty}\left(V_{m_{j}}(K)\right)^{1 / p_{j}}
$$

the transfinite diameter of $K$. In the one-dimensional case $d$ coincides with Fekete's transfinite diameter. Answering a question asked by Siciak in [12] (see also [7]), Zaharjuta [21] has shown that for any compact set in $\mathbf{C}^{n}$ the sequence $\left(V_{m_{j}}(K)\right)^{1 / p_{j}}$ converges as $j \rightarrow \infty$ and hence "lim sup" in the definition of $d(K)$ can be replaced by "lim". The transfinite diameter can also be characterized in terms of Tchebysheff constants (see $[\mathbf{2 1}]$ ).

We will need the following lemma.

Lemma 3.1. Let $\Omega$ be an open set in $\mathbf{C}^{n}$ and let $K: \Omega \rightarrow \operatorname{Comp} \mathbf{C}^{m}$ be an upper semicontinuous mapping. Define $u(z)=\log d(K(z)), z \in \Omega$. Then $u$ is locally bounded from above and $u^{*}(z)=-\infty$ if and only if $K(z)$ is pluripolar.

PROOF. Fix $z_{0} \in \Omega$. It is clear that if $\lambda>0$ and $E$ is a compact set in $\mathbf{C}^{m}$, then $d(\lambda E)=\lambda^{m} d(E)$. Hence we may assume without loss of generality that $K\left(z_{0}\right)$ is contained in the open unit ball $B \subset \mathbf{C}^{m}$. Upper semicontinuity of $K$ implies that there exists a neighborhood $U$ of $z_{0}$ such that for each $z \in U, K(z) \subset B$. Consequently $u \leq 0$ in $U$.

For any compact set $E \subset B$ define

$$
T(E)=\left(\sup \left\{\Phi_{E}(z):\|z\|=1\right\}\right)^{-1},
$$

where $\Phi_{E}$ is the Siciak extremal function (see e.g. $[\mathbf{1 2}, \mathbf{1 3}]$ ), that is

$$
\Phi_{E}(z)=\sup \left\{|p(z)|^{1 / \operatorname{deg} p}\right\},
$$

where the supremum is taken over all polynomials $p$ such that $|p| \leq 1$ on $E$ and $\operatorname{deg} p \geq 1$. It can be shown that

(a) $T(E)=0$ if and only if $E$ is pluripolar (see [13]).

(b) If $E_{1} \supset E_{2} \supset E_{3} \supset \cdots$ is a sequence of compact sets, then

$$
\left.T\left(\bigcap_{j=1}^{\infty} E_{j}\right)=\lim _{j \rightarrow \infty} T\left(E_{j}\right) \quad \text { (see }[\mathbf{1 3}]\right) .
$$


(c) There are positive constants $A$ and $\delta$ such that for any compact set $E$ contained in $B, n^{-1 / 2} T(E) \leq d(E) \leq A T(E)^{\delta}$ (see $[8]$ ).

The properties (b) and (c) imply that if $d(K(z))=0, u^{*}(z)=u(z)=-\infty$. Combining this with (a) and (c) we obtain the lemma.

In the proof of Theorem 3 we will use the following characterization of unisolvent sets (see [12]).

LEMMA 3.2. For every compact set $K \subset \mathbf{C}^{n}$ the following conditions are equivalent:

(a) $K$ is unisolvent for polynomials of degree $\leq j$.

(b) $V_{i}(K) \neq 0$ for $i=1,2, \ldots, m_{j}$.

(c) $V_{m_{j}}(K) \neq 0$.

4. Approximation lemmata for plurisubharmonic functions. Let $\Omega$ be an open subset of $\mathbf{C}^{n}$. By $\operatorname{PSH}(\Omega)$ we will denote the family of all plurisubharmonic functions on $\Omega$ (including $-\infty$ ) and by $P\left(\mathbf{C}^{n}\right)$ the family of all polynomials of $n$ complex variables.

Define $\operatorname{PSH}_{0}(\Omega)$ as the family of all functions $u \in \operatorname{PSH}(\Omega)$ which can be written in the form $u=\sup \left\{\alpha_{f} \log |f|: f \in \mathcal{F}\right\}$ for some $\mathcal{F} \subset P\left(\mathbf{C}^{n}\right)$ and $\left(\alpha_{f}\right)_{f \in \mathcal{F}} \subset\{t \in$ $\mathbf{R}: t>0\}$. In view of the following lemma we may restrict ourselves to countable families $\mathcal{F}$ in the definition of $\mathrm{PSH}_{0}$.

LEMMA 4.1. If $\mathcal{F} \subset \mathcal{C}(\Omega, \mathbf{R})$, then there is a countable family $\mathcal{F}_{0} \subset \mathcal{F}$ such that for all $z \in \Omega$

$$
\sup \{f(z): f \in \mathcal{F}\}=\sup \left\{f(z): f \in \mathcal{F}_{0}\right\} .
$$

Proof. We have

$$
\left\{(z, t) \in \Omega \times \mathbf{R}: \sup _{f \in \mathcal{F}} f(z)>t\right\}=\bigcup_{f \in \mathcal{F}}\{(z, t) \in \Omega \times \mathbf{R}: f(z)>t\} .
$$

All sets that appear in the equality are open, so in view of the Lindelöf property there exists a countable family $\mathcal{F}_{0} \subset \mathcal{F}$ such that

$$
\left\{(z, t) \in \Omega \times \mathbf{R}: \sup _{f \in \mathcal{F}} f(z)>t\right\}=\bigcup_{f \in \mathcal{F}_{0}}\{(z, t) \in \Omega \times \mathbf{R}: f(z)>t\}
$$

which proves the lemma.

Lemma 4.2 (SEe [13, TheOREM 2.13]). Let $g \in \operatorname{PSH}\left(\mathbf{C}^{n}\right)$ such that

$$
\sup _{z \in \mathbf{C}^{n}}\{g(z)-\log (1+\|z\|)\}<+\infty .
$$

Then there is a locally bounded from above sequence $\left\{g_{m}\right\}_{m \geq 1} \subset \mathrm{C}^{\infty} \cap \mathrm{PSH}_{0}\left(\mathbf{C}^{n}\right)$ such that $g(z)=\lim _{m \rightarrow \infty} g_{m}(z), z \in \mathbf{C}^{n}$.

LEMMA 4.3. Let $P_{0}$ and $P_{1}$ be closed polynomial polyhedra in $\mathbf{C}^{n}$ such that $P_{0} \subset \operatorname{int} P_{1}$. If $u \in L^{\infty} \cap \operatorname{PSH}\left(\operatorname{int} P_{1}\right)$, then there is a sequence $\left\{u_{m}\right\}_{m \geq 1} \subset$ $\mathrm{PSH}_{0}\left(\right.$ int $\left.P_{0}\right)$ which is locally bounded from above and such that

$$
u(z)=\lim _{m \rightarrow \infty} u_{m}(z), \quad z \in \operatorname{int} P_{0} .
$$

PROOF. Take $f_{1}, \ldots, f_{k} \in P\left(\mathbf{C}^{n}\right)$ such that

$$
P_{1}=\left\{z \in \mathbf{C}^{n}:\left|f_{j}(z)\right| \leq 1, j=1, \ldots, k\right\} .
$$


We can choose $\theta \in(0,1)$ such that $P_{0} \subset$ int $P_{\theta}$ where $P_{\theta}=\left\{z \in \mathbf{C}^{n}:\left|f_{j}(z)\right| \leq \theta\right.$, $j=1, \ldots, k\}$. Clearly it is enough to show the lemma for $P_{\theta}$ instead of $P_{0}$. There are positive numbers $A$ and $B$ such that $v=A(u-B)<0$ on $P_{1}$ and $d v>\log \theta$ on $P_{\theta}$ where $d=\max \left\{\operatorname{deg} f_{1}, \ldots, \operatorname{deg} f_{n}\right\}$. Define $w(z)=\max \left\{(1 / d) \log \left|f_{j}(z)\right|: j=\right.$ $1, \ldots, k\}$ and

$$
g(z)= \begin{cases}\max \{v(z), w(z)\}, & z \in \operatorname{int} P_{1}, \\ w(z), & z \in \mathbf{C}^{n} \backslash \operatorname{int} P_{1} .\end{cases}
$$

Then $g \in \operatorname{PSH}\left(\mathbf{C}^{n}\right)$ satisfies $(*)$. Moreover $g=v$ on $P_{\theta}$. Let $\left\{g_{m}\right\}$ be the sequence from Lemma 2 and let $g_{m}=\sup _{f \in \xi_{m}}\left\{\alpha_{f} \log |f|\right\}$, where $\mathcal{F}_{m} \subset P\left(\mathbf{C}^{n}\right)$ and $\alpha_{f}>0$ for each $f \in \mathcal{F}_{m}$. Define

$$
u_{m}=\sup _{f \in \mathcal{F}_{m}} \frac{\alpha_{f}}{A} \log \left|f \exp \frac{A B}{\alpha_{f}}\right| .
$$

By Lemma $2 u=\lim u_{m}$ on $P_{\theta}$.

LEMMA 4.4. Let $\left(u_{m}\right)_{m \geq 1}$ be a locally bounded from above sequence in $\operatorname{PSH}(\Omega)$ such that $u=\limsup _{m \rightarrow \infty} u_{m} \in \operatorname{PSH}(\Omega)$. If $K$ is a compact subset of $\Omega$, then $\sup u(K)=\lim \sup _{m \rightarrow \infty}\left(\sup u_{m}(K)\right)$.

PROOF. Since $u$ is upper semicontinuous there exists $z \in K$ such that

$$
\sup u(K)=u(z)=\limsup _{m \rightarrow \infty} u_{m}(z) \leq \limsup _{m \rightarrow \infty}\left(\sup u_{m}(K)\right) \text {. }
$$

The opposite inequality follows from Hartogs' Lemma (see e.g. [19, p. 76]). (If $\varepsilon>0$, the set $\{u<\sup u(K)+\varepsilon / 2\}$ is open and contains $K$, so-in view of Hartogs' Lemma - there exists $j_{0}$ such that for all $j>j_{0}$ we have $\sup u_{j}(K) \leq \sup u(K)+\varepsilon$.)

5. Joint spectra and ASV functions. In this section we shall prove Theorem 1. First of all notice that the second conclusion of the theorem is quite easy to verify. For if $A$ is commutative, then

$$
\begin{aligned}
\sigma_{A}\left(a_{1}, \ldots, a_{m}\right) & =\sigma_{A}^{L}\left(a_{1}, \ldots, a_{m}\right)=\sigma_{A}^{R}\left(a_{1}, \ldots, a_{m}\right) \\
& =\left\{\left(\phi\left(a_{1}\right), \ldots, \phi\left(a_{m}\right)\right): \phi \in \mathcal{M}\right\},
\end{aligned}
$$

where $\mathcal{M}$ is the maximal ideal space of $A$.

Let $\Omega_{1}$ be an open subset of $\Omega$ and let $u$ be a plurisubharmonic function on an open neighbourhood of the set $\left\{(z, w) \in \Omega_{1} \times \mathbf{C}^{m}: w \in \sigma_{A}(f(z))\right\}$. Then

$$
\begin{aligned}
v(z) & =\sup \left\{u(z, w): w \in \sigma_{A}(f(z))\right\} \\
& =\sup \left\{u\left(z, \phi\left(f_{1}(z)\right), \ldots, \phi\left(f_{m}(z)\right)\right): \phi \in \mathcal{M}\right\} .
\end{aligned}
$$

The mapping $z \rightarrow\left(z, \phi\left(f_{1}(z)\right), \ldots, \phi\left(f_{m}(z)\right)\right)$ is holomorphic, hence $v$ is the supremum of a family of plurisubharmonic functions. Furthermore, as $\sigma_{A}: A^{m} \rightarrow$ Comp $\mathbf{C}^{m}$ is continuous (see e.g. [11]) the function $v$ is upper semicontinuous and hence plurisubharmonic.

The noncommutative case has to be dealt with separately. If we prove the first statement of Theorem 1 for the joint left spectrum, the result will follow because $\sigma_{A}=\sigma_{A}^{L} \cup \sigma_{A}^{R}$ and $\sigma_{A}^{R}=\sigma_{\tilde{A}}^{L}$, where $\tilde{A}$ is obtained from $A$ by 'reversing' multiplication (i.e. by defining a new product of two elements $a$ and $b$ as $b a$ ). Let $B_{m}=\left\{a \in A^{m}: \sigma_{A}^{L}(a) \neq \varnothing\right\}$. By modifying the proof of Newburgh's Theorem (see e.g. $[\mathbf{1 1}]$ ) we show the following (see also $[\mathbf{4}$, p. 93]). 
LEMMA 5.1. For each positive integer $m$ the mapping $\sigma_{A}^{L}: B_{m} \rightarrow \operatorname{Comp~}^{m}$ is upper semicontinuous.

Proof. Suppose the lemma is false. There exist a neighbourhood $V$ of 0 in $\mathbf{C}^{m}$, a point $x=\left(x_{1}, \ldots, x_{m}\right) \in B_{m}$ and sequences $\left\{a_{n}\right\} \subset B_{m}$ and $\left\{z_{n}\right\} \subset \mathbf{C}^{m}$ such that $\left\|a_{n}-x\right\|<1 / n, z_{n} \in \sigma_{A}^{L}\left(a_{n}\right) \backslash\left(\sigma_{A}^{L}(x)+V\right)$ and $\left\|a_{n}\right\| \leq\|x\|+1 / n$. Since $\sigma_{A}^{L}(x) \subset \sigma_{A}\left(x_{1}\right) \times \cdots \times \sigma_{A}\left(x_{m}\right)$ and $\left(y_{1}, \ldots, y_{m}\right) \rightarrow \sigma\left(y_{1}\right) \times \cdots \times \sigma\left(y_{m}\right)$ is upper semicontinuous by Newburgh's Theorem, the sequence $\left\{z_{n}\right\}$ is bounded. Passing to a subsequence, if necessary, we may assume that $\left\{z_{n}\right\}$ is convergent. Let $\xi=\lim z_{n}$. Clearly $\xi \notin\left(\sigma_{A}^{L}(x)+V\right)$. Hence $\xi \notin \sigma_{A}^{L}(x)$. Consequently there are $y_{1}, \ldots, y_{m} \in A$ such that

$$
e=\sum_{i=1}^{n} y_{i}\left(x_{i}-\xi_{i} e\right)
$$

where $\xi=\left(\xi_{1}, \ldots, \xi_{m}\right)$. Let $a_{n}=\left(a_{n 1}, \ldots, a_{n m}\right)$ and $z_{n}=\left(z_{n 1}, \ldots, z_{n m}\right)$. The set of invertible elements of $A$ is open, so replacing $\left(x_{i}-\xi_{i} e\right)$ by $\left(a_{n i}-z_{n i} e\right.$ ) (for large $n)$ we get an invertible element $\sum_{i=1}^{m} y_{i}\left(a_{n i}-z_{n i} e\right)$ which is impossible as $z_{n} \in \sigma_{A}^{L}\left(a_{n}\right)$.

LEMMA 5.2. Let $A$ be a Banach algebra with unit and let $S_{m}=\left\{x \in A^{m}\right.$ : $\sigma_{A}^{L}(g(x))=g\left(\sigma_{A}^{L}(x)\right)$ for all polynomials $g$ of $n$ complex variables $\}$.

Let $\Omega$ be an open subset of $\mathbf{C}^{n}$ and let $f: \Omega \rightarrow S_{m}$ be holomorphic. If $P$ is a closed polynomial polyhedron in $\mathbf{C}^{n}$ such that $\sigma_{A}^{L}(f(z)) \subset$ int $P$ for all $z \in \Omega$ and $u \in \mathrm{PSH}($ int $P)$, then the function

$$
v(z)=\sup \left\{u(w): w \in \sigma_{A}^{L}(f(z))\right\}
$$

is plurisubharmonic in $\Omega$. (Note that $S_{m} \subset B_{m}$.)

ProOF. Fix $z_{0} \in \Omega$. The polynomially convex hull of $\sigma_{A}^{L}\left(f\left(z_{0}\right)\right)$ is a compact subset of int $P$. Take closed polynomial polyhedra $P_{0}$ and $P_{1}$ such that

$$
\sigma_{A}^{L}\left(f\left(z_{0}\right)\right) \subset \operatorname{int} P_{0} \subset P_{0} \subset \operatorname{int} P_{1} \subset P_{1} \subset \operatorname{int} P .
$$

By Lemma 5.1 there is a neighbourhood $W$ of $z_{0}$ such that $\sigma_{A}^{L}(f(z)) \subset$ int $P_{0}$ for all $z \in W$. Moreover, upper semicontinuity of $u$ implies that $u$ is upper bounded on $P_{1}$. By Lemma 4.4 and the fact that $u=\lim _{j \rightarrow \infty} \max (u,-j)$ we may assume that $u \in L^{\infty}\left(P_{1}\right)$. Lemmata $4.1,4.3$ and 4.4 imply that it is enough to consider $u$ of the form $u=\alpha \log |g|$, where $\alpha>0$ and $g \in P\left(\mathbf{C}^{m}\right)$. Note that the values of $f$ lie in $S_{m}$. Thus by Vesentini's Theorem $[\mathbf{1 6}, \mathbf{1 7}]$ the function $z \rightarrow r(h(z))$ (where $r$ denotes the spectral radius and $h=g \circ f)$ is logarithmically plurisubharmonic. Hence $v \in \operatorname{PSH}(W)$.

Proof OF THEOREM 1 . Let $\Omega_{1}$ be an open subset of $\Omega$ and let $G$ be an open set in $\mathbf{C}^{n} \times \mathbf{C}^{m}$ such that

$$
\{z\} \times \hat{\sigma}_{A}^{L}(f(z)) \subset G \text { for all } z \in \Omega_{1} .
$$

Suppose $u \in \operatorname{PSH}(G)$. If $g\left(z_{1}, \ldots, z_{n}\right)=\left(z_{1} e, \ldots, z_{n} e\right)$, then

$$
\{z\} \times \sigma_{A}^{L}(f(z))=\sigma_{A}^{L}(g(z), f(z)) \stackrel{\text { def }}{=} s(z)
$$

and

$$
\{z\} \times \hat{\sigma}_{A}^{L}(f(z))=\hat{s}(z) .
$$


Fix $z_{0} \in \Omega_{1}$. Take a closed polynomial polyhedron $P \subset G$ such that $\hat{s}\left(z_{0}\right) \subset$ int $P$. By Lemma 5.1 there is a neighbourhood $W$ of $z_{0}$ such that for each $z \in W, s(z) \subset$ int $P$ and hence $\hat{s}(z) \subset$ int $P$. Define

$$
v(z)=\sup \left\{u(z, w): w \in \hat{\sigma}_{A}^{L}(f(z))\right\}, \quad z \in \Omega_{1} .
$$

Then

$$
v(z)=\sup \{u(t): t \in \hat{s}(z)\}, \quad z \in \Omega_{1} .
$$

But int $P$ is a Runge domain, so $\hat{s}(z)$ coincides with the $\operatorname{PSH}($ int $P$ )-hull of $s(z)$ for each $z \in W$. Therefore for $z \in W$ we have

$$
v(z)=\sup \{u(t): t \in s(z)\} .
$$

According to Harte's Spectral Mapping Theorem (see [4, pp. 105-106]) if $x \in A^{m}$ is a commuting system of elements, then $x \in S_{m}$. Thus Lemma 5.2 implies that $v \in \operatorname{PSH}(W)$. Since plurisubharmonicity is a local property, the above shows that $v \in \operatorname{PSH}\left(\Omega_{1}\right)$.

6. Pluripolar sets and ASV functions. In this section we prove Theorems 2,3 and 4 .

PROOF OF THEOREM 2. Observe that

$$
\begin{aligned}
\log d(K(z)) & =\limsup _{j \rightarrow \infty}\left(1 / p_{j}\right) \log V_{m_{j}}(K(z)) \\
& =\limsup _{j \rightarrow \infty} \sup \left\{\left(1 / p_{j}\right) \log |V(\xi)|: \xi \in[K(z)]^{m_{j}}\right\} \\
& =\limsup _{j \rightarrow \infty} u_{j}(z) .
\end{aligned}
$$

From Theorem 2.4(b) and the definition of ASV-functions it follows that $u_{j} \in$ $\operatorname{PSH}(\Omega)$. Therefore our theorem follows from Lemma 3.1.

PROOF OF THEOREM 3. Suppose that the set $A=\{z \in \Omega$ : ord $K(z)<+\infty\}$ is not pluripolar. As in the proof of the previous theorem we can show that the function $u_{j}(z)=\log V_{m_{j}}(K(z)), z \in \Omega$, is plurisubharmonic. Lemma 3.2 implies that $A=\bigcup_{j}\left\{z \in \Omega: u_{j}(z)=-\infty\right\}$. Countable unions of pluripolar sets are pluripolar, and hence there is an integer $p_{0}$ such that $u_{p_{0}} \equiv-\infty$. By Lemma 3.2 we have $u_{k} \equiv-\infty$ for all $k \geq p_{0}$. Hence $p_{0}$ can be chosen so that $u_{p} \not \equiv-\infty$, where $p=p_{0}-1$. Define $F=\left\{z \in \Omega: u_{p}(z)=-\infty\right\}$. Obviously $p$ and $F$ have the required properties.

PROOF OF THEOREM 4. As in the definition of Fekete's transfinite diameter we may define for any compact set $K \subset \mathbf{C}^{m}$

$$
\delta_{j}(K)=\sup \left\{\prod_{p<q}\left\|z_{p}-z_{q}\right\|^{2 /(j-1) j}: z_{1}, \ldots, z_{j} \in K\right\}
$$

for $j=1,2, \ldots$. Exactly as in the one-dimensional case it follows from Theorem 2.4 that $z \rightarrow \log \delta_{j}(K(z))$ is plurisubharmonic. Using the same reasoning as for $m=1$ (see $[1$, p. 66, Theorem $1 ; 2 ; 10$, Theorem 3.11$]$ ) we can prove that either $\{z \in$ $\Omega: \# K(z)<\infty\}$ is pluripolar or there is a positive integer $p$ such that $\# K(z) \leq p$ for all $z \in \Omega$. Let us take the smallest $p$ which has this property. By Theorem 2.3 (see also $[\mathbf{1 0}$, Corollary 3.10]) $K$ is continuous and hence $F=\{z \in \Omega: \# K(z)<p\}$ 
is a closed subset of $\Omega$. We have to show that $F$ is an analytic subset of $\Omega$ and we have to construct the function $H$.

Let $\alpha: \mathbf{C}^{m} \rightarrow \mathbf{C}$ be a $\mathbf{C}$-linear mapping. For $z \in \Omega \backslash F$ define

$$
f_{\alpha}(z)=\prod_{i \neq j} \alpha\left(v_{i}(z)-v_{j}(z)\right),
$$

where $K(z)=\left\{v_{1}(z), \ldots, v_{p}(z)\right\}$. If $z \in F$, let $f_{\alpha}(z)=0$. Since $K$ is continuous, the function $f_{\alpha}$ is continuous at every point of $F$. Moreover, in view of Theorem 2.3 (and Example 2.2) for each $z_{0} \in \Omega \backslash F$ there exists a neighbourhood $W$ of $z_{0}$ and holomorphic functions $v_{1}, v_{2}, \ldots, v_{p}: W \rightarrow \mathbf{C}$ such that $K(z)=\left\{v_{1}(z), \ldots, v_{p}(z)\right\}$ for all $z \in W$. Hence $f_{\alpha}$ is holomorphic on $\Omega \backslash F$. Rado's Theorem implies that $f_{\alpha}$ is holomorphic on $\Omega$.

Let us take a system of linear functionals $\alpha_{1}, \alpha_{2}, \ldots, \alpha_{r}$ on $\mathbf{C}^{m}$ such that each subsystem of $m$ elements forms a linearly independent set and

$$
r=\frac{1}{2}(m-1) p(p-1)+1 \text {. }
$$

We claim that

$$
F=\bigcap_{j=1}^{r}\left\{z \in \Omega: f_{\alpha_{j}}(z)=0\right\} .
$$

There are only $\frac{1}{2} p(p-1)$ distinct pairs $(i, j)$ such that $1 \leq i<j \leq p$. Suppose $z \in \Omega \backslash F$ and $f_{\alpha_{1}}(z)=\cdots=f_{\alpha_{r}}(z)=0$. Then there exists a pair $(i, j)$ and $m$ distinct values of $k$ for which $\alpha_{k}\left(v_{i}(z)-v_{j}(z)\right)=0$. Hence $v_{i}(z)=v_{j}(z)$. This contradicts our assumption that $z \notin F$. Therefore $f_{\alpha_{1}}(z)=\cdots=f_{\alpha_{r}}(z)=0$ if and only if $z \in F$.

In a similar way we may prove the existence of $H$. For $z \in \Omega \backslash F$ and $w \in \mathbf{C}^{m}$ define

$$
h_{k}(z, w)=\prod_{j=1}^{p} \beta_{k}\left(w-v_{j}(z)\right),
$$

where $k=1,2, \ldots,(m-1) p+1$ and the $\beta_{k}$ 's are $\mathbf{C}$-linear functionals on $\mathbf{C}^{m}$ chosen so that every set of $m$ of these functionals is linearly independent. Theorem 2.3 implies analyticity of $h_{k}$ on $(\Omega \backslash F) \times \mathbf{C}^{m}$. As a consequence of Riemann's extension theorem, for each $h_{k}$ there is a holomorphic function $H_{k}: \Omega \times \mathbf{C}^{m} \rightarrow \mathbf{C}$ that coincides with $h_{k}$ on $(\Omega \backslash F) \times \mathbf{C}^{m}$. Clearly if $z \in \Omega \backslash F$, then $w \in K(z)$ if and only if $H_{k}(z, w)=0$ for all $k=1,2, \ldots,(m-1) p+1$. Indeed, there are only $p$ distinct points in $K(z)$. So, if $H_{k}(z, w)=0$ for $k=1,2, \ldots,(m-1) p+1$, then there exists a point $w_{0} \in K(z)$ and $m$ distinct values of $k$ for which $\beta_{k}\left(w-w_{0}\right)=0$. Hence $w=w_{0}$.

Now assume that $z_{0} \in F$. We may proceed as in $[\mathbf{1 0}, \mathrm{p} .40]$. Take a sequence $\left\{z_{j}\right\} \subset \Omega \backslash F$ such that $z_{j} \rightarrow z_{0}$. Then $H\left(z_{0}, w_{0}\right)=0$ if and only if there exists a sequence $w_{j} \rightarrow w_{0}$ with $w_{j} \in K\left(z_{j}\right)$. This, in turn, is equivalent to the fact that $w_{0} \in K\left(z_{0}\right)$, since $K$ is continuous.

\section{REFERENCES}

1. B. Aupetit, Propriétés spectrales des algèbres de Banach, Lecture Notes in Math., vol. 735, Springer-Verlag, 1979.

2. Math. 44 (1982), 18-60. 
3. R. Engelking, General topology, Polish Scientific Publishers, Warsaw, 1977.

4. R. E. Harte, Spectral mapping theorems, Proc. Royal Irish Acad. 72A (1972), 89-107.

5. K. Kuratowski, Les fonctions semi-continues dans l'espace des ensembles fermés, Fund. Math. 18 (1932), 148-159.

6. __ Operations on semi-continuous set-valued mappings, Seminari 1962-1963, Ist. Naz. Alta Mat. Roma, vol. II, Ediz. Cremonese, Roma, 1965, pp. 449-461.

7. F. Leja, Problèmes a resoudre poses à la conference, Colloq. Math. 7 (1959), 151-153.

8. N. Levenberg and B. A. Taylor, Comparison of capacities in $\mathbf{C}^{n}$, preprint, 1983.

9. K. Oka, Note sur les familles des fonctions analytiques multiformes etc., J. Sci. Hiroshima Univ. 4 (1934), 93-98.

10. T. J. Ransford, Analytic multivalued functions, Dept. of Math., Univ. of Cambridge, Cambridge, 1983.

11. C. E. Rickart, General theory of Banach algebras, Van Nostrand, Princeton, N. J., 1966.

12. J. Siciak, On some extremal functions and their applications in the theory of analytic functions of several complex variables, Trans. Amer. Math. Soc. 105 (1962), 322-357.

13. __ Extremal plurisubharmonic functions and capacities in $\mathbf{C}^{n}$, Sophia Kokyuroku in Mathematics 14, Sophia University, Tokyo, 1982.

14. Z. Słodkowski, On the subharmonicity of the capacity of the spectrum, Proc. Amer. Math. Soc. 81 (1981), 243-249.

15. __ Analytic set-valued functions and spectra, Math. Ann. 256 (1981), 363-386.

16. E. Vesentini, On the subharmonicity of the spectral radius, Boll. Un. Mat. Ital. 4 (1968), 427-429.

17. __ Maximum theorems for spectra, Essays on Topology and Related Topics, Mémoires dédiés à Georges de Rham, Springer-Verlag, 1970, pp. 111-117.

18. __ Carathéodory distances and Banach algebras, Adv. Math. 47 (1983), 50-73.

19. V. S. Valdimirov, Methods of the theory of functions of several complex variables, The MIT Press, Cambridge, Mass. and London, 1966.

20. H. Yamaguchi, Sur une uniformité des surfaces constantes d'une fonction entière de deux variables complexes, J. Math. Kyoto Univ. 13-3 (1973), 417-433.

21. V. Zaharjuta, Transfinite diameter, Cebysev constants and capacity for compact a in $\mathrm{C}^{n}$, Math. USSR Sb. 25 (1975), 350-364.

22. A. Zraibi, Sur les fonctions analytiques multiformes, Thesis, Université Laval, Quebec, 1983.

Department of Mathematics, University College Dublin, Belfield, Dublin 4, IRELAND 